\title{
TEM and STEM Studies of the Atomic Structure and Chemistry of Ferritin in Human Liver Biopsies
}

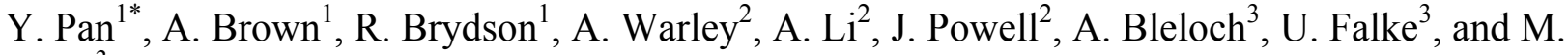 \\ Falke $^{3}$
}

${ }^{1}$ Institute for Materials Research, University of Leeds, Leeds LS2 9JT, UK

${ }^{2}$ The Rayne Institute, St Thomas' Hospital, London SE1 7EH, UK

${ }^{3}$ SuperSTEM, Daresbury Laboratories, Warrington, WA4 4D, UK

During the daily human iron cycle any excess iron is temporarily stored in the liver by ferritin molecules before being cycled back into the body. The ferritin molecule (12nm in diameter) consists of a crystalline hydrated iron oxide core $(6 \mathrm{~nm}$ in diameter) and an organic shell that facilitates the movement of iron in and out of the core. Several groups have presented different structural models of extracted ferritin cores from various sources, such as horse spleen or human brain, but none of them has been widely accepted $[1,2,3]$. The work we present here is the first work to characterise human liver ferritin cores in-situ of extracted human liver tissue by using analytical electron microscopy.

We present new data using aberration-corrected scanning transmission electron microscopy (SuperSTEM) operating at $100 \mathrm{keV}$. Although there is little or no electron beam damage issues that are referred to in the literature, beam damage was clearly observed in the experiments we have conducted. In order to get a true representation of the crystal structure, a safe electron dose has been established in our first set of experiments. Data and images at this dose show that the ferritin core crystallises predominantly in the ferrihydrite structure, which should only contain Fe ${ }^{3+}$. FIG. 1 shows a high angle annular dark field image (HAADF image) of (a) a region of isolated ferritin cores within extracted tissue and (b) individual ferritin cores revealing a polycrystalline structure within the individual core.

Our earlier TEM work in some regions of the cell suggests the presence of a low energy $\mathrm{Fe}^{2+}$ shoulder in the $\mathrm{Fe}_{3}$-edge measured by EELS. These results were obtained from both individual ferritin cores and clusters of cores in both the unstained (least altered) high iron loaded (haemochromatosis) and normal livers [5]. Valence quantification [6, 7] suggests that ferritin molecules in these livers can contain up to $35 \% \mathrm{Fe}^{2+}$. Our current TEM EELS studies of ferritin suggest that the Fe valence of individual cores varies depending on the position within different regions of the cell. The results obtained at an energy resolution $\sim 0.7 \mathrm{eV}$, shown in FIG. 2 , indicate different $\mathrm{Fe}^{2+} / \mathrm{Fe}^{3+}$ ratios at the edge and in the centre of a cluster of ferritin cores- evidenced by the chemical shift of the $\mathrm{Fe} \mathrm{L}_{3}$-edge. We are currently establishing if the beam conditions for $\mathrm{TEM} / \mathrm{EELS}$ is of a safe dose for valence measurement.

Further EELS and EDX measurements of clusters and isolated ferritin molecules in the tissue will investigate the intracellular variation in Fe valency, which is thought to be affected by the phosphorus content. This phosphorus content is also undergoing investigation. 
Reference:

[1] J. Cowley et al., Journal of Structural Biology. 131 (2000) 210.

[2] C. Quintana et al., Cellular and Molecular Biology. 4 (2000) 807.

[3] C. Quintana et al., Journal of Structural Biology. 147 (2004) 166.

[4] K. Towe and W. Bradley, Journal of Colloid and Interface Science. 24 (1967) 384.

[5] A. P. Brown et al., Inst. Phys. Conf. Ser. 179 (2003) 83.

[6] C. C. Calvert et al., Inst. Phys. Conf. Ser. 168 (2001) 251.

[7] Y. Pan et al., European Microscopy Congress 2004 proceedings. LS06 P07 (2004) 1.
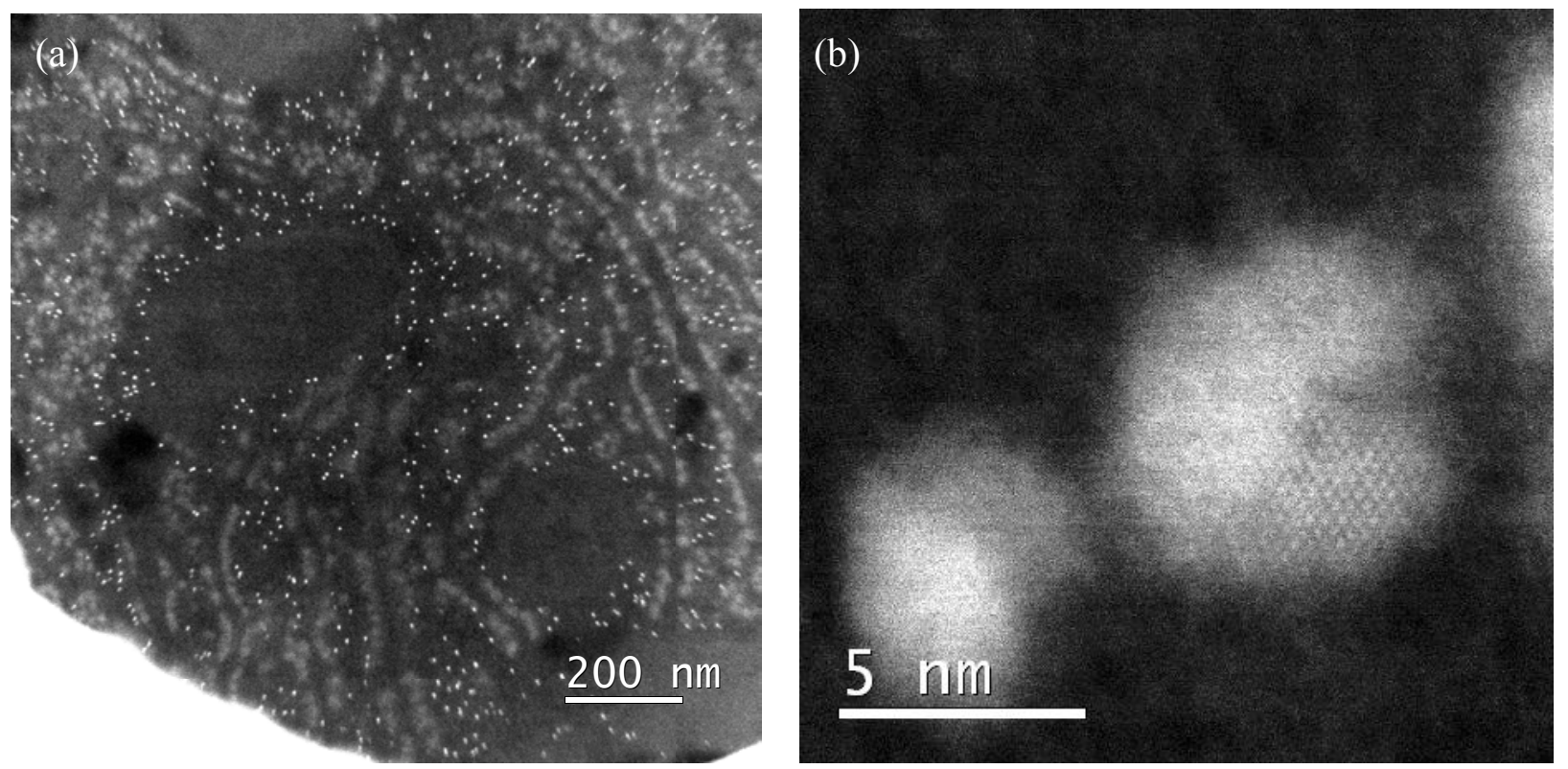

FIG. 1. HAADF images of (a) isolated ferritin cores within extracted tissue; (b) individual ferritin molecules.
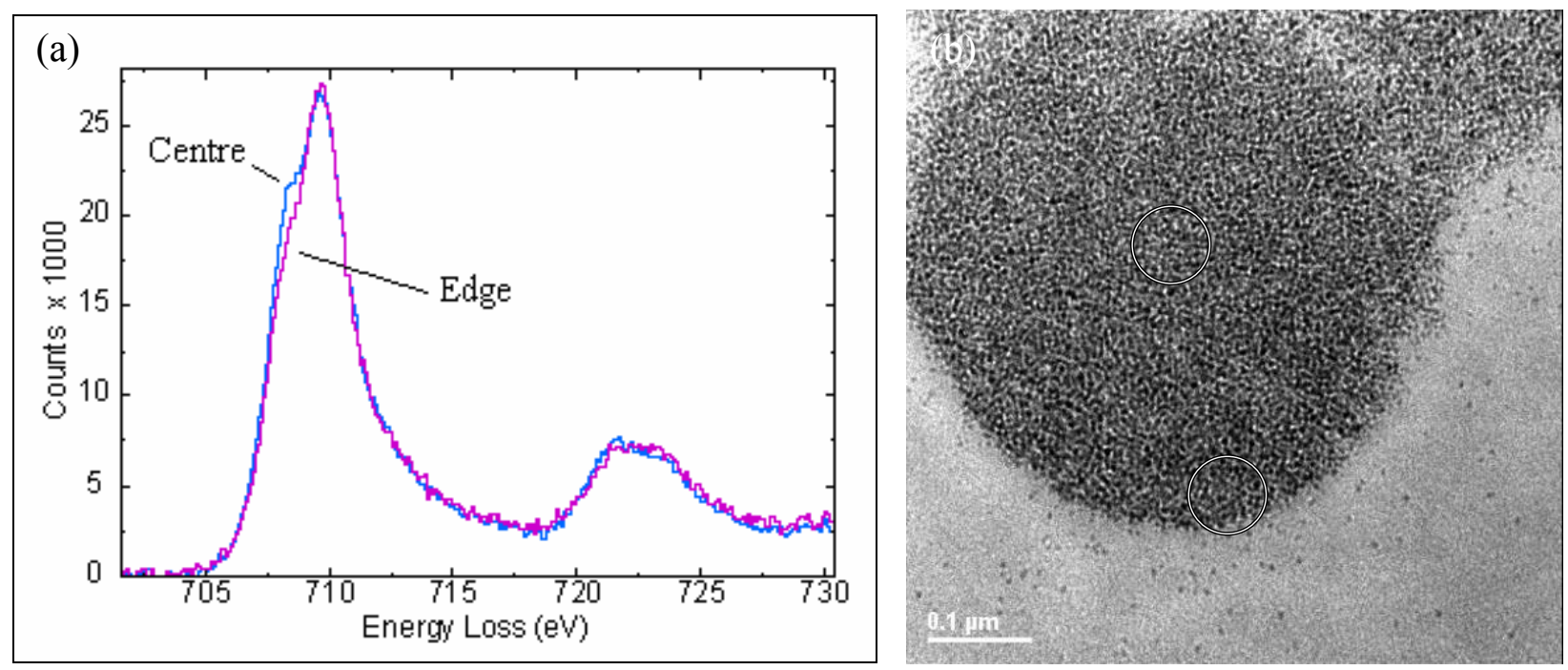

FIG. 2. (a) A typical TEM EELS Fe $\mathrm{L}_{2,3}$-edge from the edge and the centre of a ferritin cluster comparison. (b) The areas from whish EELS spectra were taken. 